\title{
Datenbankgruppen vorgestellt - Das Institut für Informationssysteme (IFIS) an der TU Braunschweig
}

Wolf-Tilo Balke

Online publiziert: 20. Mai 2010

(C) Springer-Verlag 2010

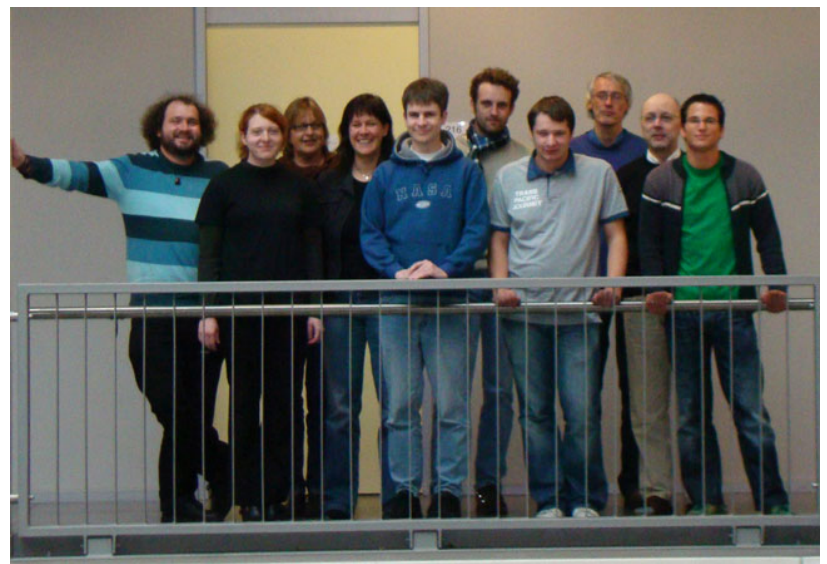

Das Institut für Informationssysteme an der TU Braunschweig gruppiert sich um den Lehrstuhl für Datenbanken und Informationssysteme, der im Sommersemester 2008 durch Prof. Wolf-Tilo Balke neu besetzt wurde. Im Wesentlichen besteht der Lehrstuhl aus 4 Arbeitsgruppen, die sich mit den Gebieten Personalisierte Datenbankanfragen, Digitale Bibliotheken, Bioinformationssysteme und GeoInformationssysteme (GIS) befassen. Zentrales Thema des Lehrstuhls ist der Bereich der Personalisierung, welcher alle Anwendungsgebiete von Informationssystemen durchdringt.

W.-T. Balke ( $\varangle)$

Institut für Informationssysteme, Mühlenpfordtstr. 23,

38106 Braunschweig, Deutschland

e-mail: balke@ifis.cs.tu-bs.de

url: www.ifis.cs.tu-bs.de

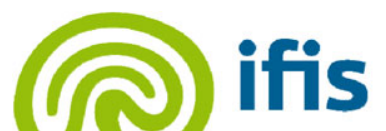

Institut für Informationssysteme Technische Universität Braunschweig

Im Kerndatenbankbereich wird deshalb die individuell angepasste Verarbeitung von Anfragen untersucht, wobei besonderes Augenmerk den Skyline Queries und Präferenzbasierten Anfragetypen gilt. Dabei geht es einerseits um Fragen der Performanz, aber auch speziell um das Problem, welche Ergebnisse für jeden individuellen Nutzer eigentlich relevant sind. Also steht hier die Einbindung von Kontextwissen, typischen Workflows und persönlichen Profilen im Vordergrund. Derzeit wird aktiv an der Repräsentation und Einbindung von Wissen in Form von Ontologien und Folksonomien in den Retrievalprozess geforscht.

Eingebettet in das Forschungszentrum L3S in Hannover profitiert die Arbeitsgruppe Digitale Bibliotheken des IFIS von der Nähe zur TIB Hannover, einer der drei nationalen Fachbibliotheken Deutschlands. Der Fokus dieser Gruppe liegt in der Entwicklung innovativer, sowie nachhaltiger Mehrwertdienste für die individuelle Informations- 
versorgung. Dabei sind insbesondere die Erschließung von personalisierten Wissensräumen, die semantisch hochwertige automatische Metadatenerzeugung, sowie die Qualitätskontrolle von großen digitalen Beständen zu erforschen.

Seit 2002 beschäftigt sich die BioinformationssystemeGruppe unter der Leitung von Dr. Silke Eckstein mit der Modellierung und Simulation biologischer Netzwerke sowie der Verwendung von Ontologien zur semantischen Integration von Datenbanken. Anwendungen liegen dabei vor allem in der Systembiologie, in der die Informatik hilft, die komplexen Stoffwechsel- und Signalübertragungsabläufe inner- halb von Zellen besser zu verstehen, zu rekonstruieren und Vorhersagen über bisher nicht erforschte Teilnetze zu treffen.

Schließlich arbeitet die GIS-Gruppe unter Führung des akademischen Direktors Dr. Karl Neumann hauptsächlich auf dem Gebiet „automatische Kartographie“ und hat in jüngster Vergangenheit z.B. die Ableitungsregeln und Signaturen für Liegenschaftskarten aus einem informellen Format in ausführbaren Code transformiert. Für die nähere Zukunft ist die Generierung von personalisierten, maßstabsabhängigen Bildschirmkarten geplant. 